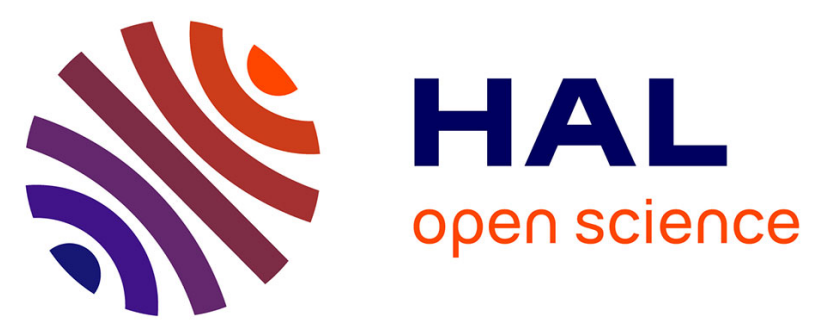

\title{
Immunoassay quantification of human insulin added to ternary parenteral nutrition containers: comparison of two methods
}

\author{
Heloise Henry, Damien Lannoy, Nicolas Simon, David Seguy, Michele \\ d'Herbomez, Christine Barthelemy, Bertrand Decaudin, Thierry Dine, Pascal \\ Odou
}

\section{To cite this version:}

Heloise Henry, Damien Lannoy, Nicolas Simon, David Seguy, Michele d'Herbomez, et al.. Immunoassay quantification of human insulin added to ternary parenteral nutrition containers: comparison of two methods. Analytical and Bioanalytical Chemistry, 2017, Analytical and bioanalytical chemistry, 409, pp.3677-3684. 10.1007/s00216-017-0311-0 . hal-02149403

\section{HAL Id: hal-02149403 \\ https: / hal.univ-lille.fr/hal-02149403}

Submitted on 6 Jun 2019

HAL is a multi-disciplinary open access archive for the deposit and dissemination of scientific research documents, whether they are published or not. The documents may come from teaching and research institutions in France or abroad, or from public or private research centers.
L'archive ouverte pluridisciplinaire HAL, est destinée au dépôt et à la diffusion de documents scientifiques de niveau recherche, publiés ou non, émanant des établissements d'enseignement et de recherche français ou étrangers, des laboratoires publics ou privés. 


\section{IMMUNOASSAY QUANTIFICATION OF HUMAN INSULIN ADDED TO TERNARY PARENTERAL NUTRITION CONTAINERS: COMPARISON OF TWO METHODS}

Authors: Héloïse Henry ${ }^{1,2}$, Damien Lannoy ${ }^{1,2}$, Nicolas Simon ${ }^{1,2}$, David Seguy ${ }^{3,4}$, Michèle D’Herbomez $^{5}$, Christine Barthélémy ${ }^{1}$, Bertrand Décaudin ${ }^{1,2}$, Thierry Dine ${ }^{1}$, Pascal Odou ${ }^{1,2}$

1. Univ. Lille, EA 7365 - GRITA - Groupe de Recherche sur les formes Injectables et les Technologies Associées, F-59000 Lille, France

2. Pharmacy Institute, CHU Lille, F-59000 Lille, France

3. University of Lille, U995 - LIRIC - Lille Inflammation Research International Center, F-59000 Lille, France

4. University Hospital of Lille, Department of Nutrition, F-59000 Lille, France

5. Immunoanalysis center, Biology and Pathology Center, CHU Lille, F-59000 Lille, France

Corresponding author:

Dr. Damien Lannoy

Faculté des Sciences Pharmaceutiques et Biologiques, EA 7365 - GRITA Laboratoire de Biopharmacie, Pharmacie Galénique et Hospitalière, 3, rue du Professeur Laguesse - B.P. 83, 59006 Lille Cedex, France.

Tel: +33 320964029

Fax: +33 (0)320959009

E-mail address: damien.lannoy@univ-lille2.fr.

Acknowledgments: the authors especially want to thank Mrs Alexandra Tavernier for her extensive revision of the final manuscript. 


\section{ABSTRACT}

Background: Adding insulin directly into infusion bags seems to be a useful method for controlling hyperglycemia in patients under ternary parenteral nutrition (TPN). Its efficacy is assessed by glycemic monitoring but few data are available on insulin stability in this situation. Among the various methods for quantifying insulin levels in human serum, the immunoassay ones seemed potentially appropriate for a TPN admixture containing high lipid concentrations. We sought to identify and validate which of two immunoassay methods was the better to quantify human insulin and consequently be adapted to studying its stability in a TPN admixture.

Methods: Two immunoassay methods to quantify recombinant human insulin were assessed in industrial TPN: an immunoradiometric assay (IRMA) and an immunoelectrochemiluminometric assay (IECMA). Validation trials for both methods were based on the accuracy profile method.

Results: Interference with immunometric assays due to the high lipidic content of TPN was eliminated through an improved preparation protocol using a bovine serum albumin (BSA) diluted in phosphate buffer saline (PBS). The relative total error of IECMA varied from 1.74 to $4.52 \%$ while it varied from -0.32 to $8.37 \%$ with IRMA. Only IECMA provided an accuracy profile with a $95 \%$ confidence interval of calculated-tolerance limits falling between the chosen acceptance limits (i.e. total error $\leq \pm 10 \%$ ).

Conclusions: IECMA combined with a BSA dilution is a simple and semi-automatic method that provides an accurate quantification of human insulin in a TPN admixture without any interference from lipids.

Keywords: human insulin, parenteral nutrition solutions, immunoassay, validation, accuracy profile 


\section{INTRODUCTION}

The aim of parenteral nutrition therapy is to supplement or cover nutritional requirements via the intravenous route when both oral and enteral intakes are insufficient or contraindicated. Ternary parenteral nutrition (TPN) corresponds to an admixture providing macronutrients (glucose, amino-acids, lipids) and micronutrients (electrolytes, vitamins and trace elements). Considering the amount of glucose infused, hyperglycemia is very common during TPN in elderly and home parenteral nutrition patients, and concerns most patients in intensive care units $[1,2]$. Hyperglycemia in both non-critically and critically ill patients receiving TPN is known to be responsible for an increase in the risk of hospital mortality [3]. Such patients may require exogenous insulin in their TPN, but in spite of the guidelines on safe practices for parenteral nutrition provided by the American Society of Parenteral and Enteral Nutrition [4], few data concerning the stability of insulin in TPN are available $[5,6]$.

Insulin concentration is currently measured by separative methods, such as high performance liquid chromatography coupled with UV detection [7-9] or size exclusion chromatography [10], which are not adapted to the complex TPN medium. Mass spectrometry-based methods, more sensitive but more expensive, require a complicated sample preparation (i.e. immunoextraction) and dedicated equipment [11]. Radiolabeled insulin $\left({ }^{125}\right.$ I-insulin) [12] is useful for studying adsorption on containers, but cannot indicate any modification in biological activity, or offer any assessment of either structural or conformational changes in insulin (from primary to quaternary structure). The presence of lipids hinders the accurate measurement of insulin concentration in all-in-one TPN admixtures regardless of the quantification method [13]. This means that other assays, capable of measuring the amount of insulin in TPN admixtures precisely, have to be carried out. Immunometric assays are routinely used today to quantify human insulin and its analogues in serum [14-16]. The 
ImmunoRadioMetric Assay (IRMA) [17] and ImmunoElectroChemiluminoMetric Assay (IECMA) [18] have shown a high level of specificity for human insulin because of their mechanism involving anti-insulin antibodies as well as their high sensitivity.

The aim of our study was to determine the ability of such techniques to quantify insulin at low concentrations in a TPN admixture, to validate them and make use of one in a stability study of insulin in a TPN admixture.

\section{MATERIALS AND METHODS}

Drugs, chemicals and reagents

Olimel $^{\mathrm{TM}}$ N7E (1.5 L bags, Baxter, Deerfield, USA) is the mixture used as TPN (containing glucose, amino acid solution, with other electrolytes and lipid emulsion). Decan ${ }^{\mathrm{TM}}$ (Aguettant, Lyon, France) and Cernevit ${ }^{\mathrm{TM}}$ (Baxter, Deerfield, USA) were used as additives to TPN. Umuline $^{\mathrm{TM}}$ rapid (100 IU/mL human insulin) was purchased from Eli-Lilly (Suresnes, France). Isotonic saline serum $(0.9 \% \mathrm{NaCl})$ was purchased from Baxter.

Sodium dihydrogen phosphate monohydrate $\left(\mathrm{NaH}_{2} \mathrm{PO}_{4}, \mathrm{H}_{2} \mathrm{O}\right)$ and sodium phosphate dibasic dodecahydrate $\left(\mathrm{Na}_{2} \mathrm{HPO}_{4}, 12 \mathrm{H}_{2} \mathrm{O}\right)$, designed for phosphate buffer saline (PBS) preparations, were purchased from Cooper (Melun, France). Hydrochloric acid ( $\mathrm{HCl} 25 \%$ ) and tris(hydroxymethyl)aminomethane (TRIS) powder were purchased from Merck Millipore (Molsheim, France). Bovine serum albumin (BSA) V-fraction was purchased from Euromedex (Souffelmeyersheim, France). Diluted human serum albumin (dHSA, Vialebex ${ }^{\mathrm{TM}}$ $40 \mathrm{mg} / \mathrm{mL}$ ) was purchased from LFB (Lille, France). Human gamma globulins (HGBO) were purchased from Sigma Aldrich (Saint Louis, USA). 


\section{Sample preparation}

Cernevit $^{\mathrm{TM}}$ was reconstituted with $5 \mathrm{~mL}$ of $0.9 \% \mathrm{NaCl}$ and gently shaken to obtain a homogenous solution. Decan ${ }^{\mathrm{TM}}$ was then directly introduced into the TPN bag. Seals were finally broken by rolling the bag onto itself to mix all nutrients of the TPN admixture. After mixing, this work medium corresponded to the TPN admixture (emulsion enriched with vitamins and trace elements). It could be stored at $5 \pm 3^{\circ} \mathrm{C}$ up to 24 hours and used for other assays.

All assays were performed at a 20,000 $\mu \mathrm{IU} / \mathrm{mL}$ (i.e. $20 \mathrm{IU} / \mathrm{L}$ ) insulin concentration, corresponding to the usual concentration in medical wards when insulin is added to TPN. Every step was carried out with calibrated laboratory materials. Samples were taken from flasks immediately after preparation without any intermediate transfer step into another container.

\section{Instrumentation}

Immunoreactive insulin concentration was determined by IRMA with the Bi-insulin IRMA ${ }^{\mathrm{TM}}$ set (Cisbio international, Paris, France) or by IECMA with the Insulin Elecsys ${ }^{\mathrm{TM}}$ assay (Roche diagnostics, Meylan, France). As calibration and measuring ranges were respectively 1 to $500 \mu \mathrm{IU} / \mathrm{ml}$ for Bi-insulin $\mathrm{IRMA}^{\mathrm{TM}}$ and 0.2 to $1000 \mu \mathrm{IU} / \mathrm{ml}$ for Insulin Elecsys ${ }^{\mathrm{TM}}$, a twostep 1/200 dilution of all samples was performed. Final samples were analyzed on two different instruments: radioactivity was determined on a Wizard 1470 Gamma coulter (CisBio, Saclay, France), while IECMA used an e601 Cobas (Roche diagnostics, Mannheim, Germany). The latter apparatus was calibrated with a Calset insulin calibrating kit and controlled with Precicontrol multimarker solutions both purchased from the same manufacturer (Roche, Mannheim, Germany). All assays were performed in glass containers (volumetric flasks or tubes) to avoid container-content interactions. 
The values are given in counts per minute (CPM) for IRMA and in $\mu \mathrm{IU} / \mathrm{mL}$ for IECMA. For all samples, IRMA values were converted into their corresponding $\mu \mathrm{IU} / \mathrm{mL}$ concentrations basing calculations on a 4-parameter calibration curve (obtained by spline function or Rodbard equation [19]), which had previously been fed into the FiaCalc ${ }^{\mathrm{TM}}$ software provided with the gamma coulter.

\section{Matrix effect}

As explained below, with immunoassays, quantification can be made at very low concentrations, thus a two-step 1/200 sample dilution was required. Both dilution steps were performed in hemolysis glass tubes using a diluent with an insignificant matrix effect (tested with both methods). Different types of diluents were selected and tested after a review of the literature with regard to immunoassays and information on the composition of calibration standards contained in commercial kits, provided by the user manuals of both methods. Both dHSA and BSA, at a concentration range of 5 to $80 \mathrm{~g} / \mathrm{L}$, were diluted in $0.9 \% \mathrm{NaCl}, 0.04 \mathrm{M}$ phosphate buffer [20], 0.04 M phosphate buffer enriched with HGBO [20], or 0.1 M TRIS buffer (prepared according to the French Pharmacopoeia $11^{\text {th }}$ edition). The tested concentrations were 5, 10, 15, 20, 25, 30, 35, $40 \mathrm{~g} / \mathrm{L}$ for dHSA and 5, 10, 20, 30, 40, 50, 60, 70, $80 \mathrm{~g} / \mathrm{L}$ for BSA. All buffers were previously adjusted to $\mathrm{pH} 7.53$ with $\mathrm{HCl}$. Diluent was prepared as follows: BSA or dHSA was first added into the chosen volume of $0.9 \% \mathrm{NaCl}$ or buffer and then gently shaken (to avoid foam generation) until total dissolution. Measurements of all diluents were made in triplicate immediately after preparation with both immunometric methods following user manual instructions to determine which diluent could be used without any matrix effect. Results are presented as means of the three values. 
To validate the assay, the preparation of calibration standard (CS) and quality control (QC) samples was performed according to the accuracy profile method. Five CS ranging from 5,000 to $40,000 \mathrm{IU} / \mathrm{mL}$ (i.e. 5, 10, 20, 30 and $40 \mathrm{IU} / \mathrm{L}$ ) and four QC concentrations $(7.5,15$, 25 and $35 \mathrm{IU} / \mathrm{L}$ ) were prepared for imprecision and linearity studies. All samples were treated identically. CS and QC were freshly prepared by spiking the TPN admixture with volumes of insulin solution (fig 1). The same preparation protocol was used for both methods.

\section{Method validation}

Validation of both methods was obtained through the accuracy profile method. Theoretical aspects of the accuracy profile are detailed by Hubert et al. [21] in the French Society of Pharmaceutical Sciences and Techniques guidelines and by Cofrac (French National Accreditation body) in the accreditation technical guide in human health SH-GTA-04 [22] to certify assays in medical biology.

Accuracy, called "total error", corresponds to the addition of systematic error (trueness) and random error (precision) obtained with QC. Each day, a blank sample (described below), five calibration standards and four QCs, prepared in triplicate were analyzed. Finally, the accuracy profile was computed at a type I risk of $5 \%$, with acceptance limits of $\pm 10 \%$, even though immunoassay techniques are usually validated at $\pm 20 \%$ [23]. Trueness is represented by relative error and precision as estimated by the expectation tolerance limits (or tolerance interval), which were established through a Student t-test $(\beta=95 \%)$.

In order to evaluate the matrix effect on immunometric reactions, blank samples (treated with the same sample preparation protocol described previously) were measured ten times with both IRMA and IECMA. The objective was to confirm the absence of any signals signifying the absence of a matrix effect linked to the TPN admixture. These blank samples were also 
essential in calculating limits of detection (LOD) and quantification (LOQ) which were established for both methods, following the International Conference on Harmonization (ICH) recommendations, from the standard derivation of 10 blank values obtained with the work medium (named $\mathrm{s}_{\mathrm{b}}$ ), as shown in Eq (1) and (2).

$\mathrm{LOD}=3.3 \times \mathrm{s} \mathrm{s}_{\mathrm{b}} / \mathrm{slope} \quad \mathrm{Eq}(1)$

$\mathrm{LOQ}=10 \times \mathrm{x} \mathrm{s}_{\mathrm{b}} /$ slope $\quad \mathrm{Eq}(2)$

Another method described in the Cofrac technical guidelines for accreditation [22] consists in defining LOQ and LOD with the signal/noise $(\mathrm{S} / \mathrm{N})$ ratio with values from the repetition of 30 blank samples. In that case:

$\mathrm{LOD}=3 \times \mathrm{S} / \mathrm{N}(3)$

$\mathrm{LOQ}=10 \times \mathrm{S} / \mathrm{N} \quad \mathrm{Eq}(4)$

All statistics and computations were obtained using Excel software (Microsoft ${ }^{\circledR}$ Office suite v2010).

To reinforce the comparison between the two methods, an analysis of variance (ANOVA) was performed, using XLSTAT (Addinsoft ${ }^{\circledR}$, Paris, France).

\section{RESULTS}

Matrix effect

Results obtained with the various diluents for the matrix effect study are reported in table 1.

\section{- IRMA}

The matrix effect in TRIS buffer was considerable (above $5.7 \mu \mathrm{IU} / \mathrm{mL}$ ) whatever the dHSA concentrations tested. BSA solution in $0.9 \% \mathrm{NaCl}$ also yielded a strong matrix effect (which actually reached a maximum of $5.37 \mu \mathrm{IU} / \mathrm{mL}$ for the highest BSA concentration) for all tested 
concentrations. The diluent giving the weakest matrix effect seemed to be BSA diluted in PBS at $40 \mathrm{~g} / \mathrm{L}$, which was then chosen as diluent for the IRMA method.

\section{- IECMA}

Whatever the nature of serum albumin, its concentration range or dissolution medium, the matrix effect obtained by IECMA never reached zero value. Indeed, when the value obtained was $<\mathrm{LOD}$, the data software did not provide concentration data. All values were close to the $0.2 \mu \mathrm{IU} / \mathrm{mL}$ LOD value. As with the IRMA method, BSA in PBS at $40 \mathrm{~g} / \mathrm{L}$ showed a weak matrix effect. This diluent was again chosen for the IECMA quantification method. 


\title{
Method validation
}

Calculations were made with experimental values provided by automated appliances after calibrations steps. Validation trials were carried out with the accuracy profile method. Both IRMA and IECMA methods showed linearity with a calculated $r^{2}>0.99$ relationship between theoretical and back-calculated concentrations over the whole concentration range (with $\mathrm{p}$ values $<0.0001$ obtained by ANOVA analysis). Results are presented in tables 2 and 3 .$$
\text { - IRMA }
$$

Limits of detection or quantification calculated with results of the IRMA method are detailed in table 2. The within-day precision (i.e. repeatability) for human insulin in TPN is less than $5.2 \%$ and between-day precision (i.e. intermediate precision) is less than $28.51 \%$ (table 3 ). As shown in figure $2 \mathrm{a}$, the accuracy profile was not validated within the acceptance limits of $\pm 10 \%$ for the defined concentration range.
\end{abstract}

\begin{abstract}
- IECMA
Both LOD and LOQ obtained with the IECMA method are presented in table 2. The withinday precision did not exceed $2.46 \%$ and the between-day precision was below $7.98 \%$ (table 3). The accuracy profile (figure 2 b) was validated at $95 \%$ with an acceptance limit of $\pm 10 \%$ of the nominal value for the concentration range indicated in table 2 .
\end{abstract}


$=$

$\ldots$ 
Table 3: Precision parameters of both methods. Both intra-day (within-day) and inter-day (between-day) precisions for quality controls are determined with relative standard deviation (\%RSD).

\begin{tabular}{|l|l|l|l|}
\hline $\begin{array}{l}\text { Quantification } \\
\text { method }\end{array}$ & $\begin{array}{l}\text { Insulin } \\
\text { concentrations } \\
\text { (IU/L) }\end{array}$ & $\begin{array}{l}\text { Within-day } \\
\text { precision (n=3) } \\
\text { (\%RSD) }\end{array}$ & $\begin{array}{l}\text { Between-day } \\
\text { precision (n=9) } \\
\text { (\%RSD) }\end{array}$ \\
\hline IRMA & 7.5 & 3.45 & 4.57 \\
& 15 & 2.68 & 5.94 \\
& 25 & 5.20 & 28.51 \\
& 35 & 3.43 & 18.79 \\
\hline IECMA & 7.5 & 2.16 & 2.51 \\
& 15 & 1.49 & 2.27 \\
& 25 & 2.46 & 7.98 \\
& 35 & 1.21 & 3.32 \\
\hline
\end{tabular}




\section{DISCUSSION}

Our results show that IECMA is more precise than IRMA in measuring human insulin concentration in TPN. To our knowledge, this is the first time that a chemiluminescent method (IECMA) has been proved capable of establishing this quantification in a TPN complex medium.

Several studies measuring insulin in TPN bags have previously been performed. The techniques used were either gamma counting of added $\mathrm{I}^{125}$ radiolabeled insulin [12] or radioimmunoanalysis (RIA) [5,6,24,25]. However, these techniques have different negative aspects. First, gamma counting is based on radiolabeled insulin whose ternary and/or quaternary structure can be modified by labeling, which means it does not reflect the real behavior of insulin in the environment. Secondly, RIA, which uses polyclonal antibodies, has a lower specificity than the IRMA or IECMA techniques, which use monoclonal antibodies [26]. Moreover, as regards the quantification of insulin in human serum, IECMA showed a lower LOQ, a larger dynamic analysis range without dilution and closer concordance during the reanalysis of a chosen sample, compared to RIA [19]. This explains why, in our case, IRMA failed to yield a precise quantification of insulin in TPN. The advantage of our technique is that it was validated in a complete TPN medium containing various different chemical species (dextrose, amino acids and lipids enriched with vitamins and trace elements) while previous studies have not been validated in such an environment. The disadvantage of the only study which has so far used ternary parenteral nutrition [5] was to quantify insulin recovery from a TPN admixture contained in PVC or EVA bags at the end of the infusion line but his work did not target the stability of insulin with only a TPN admixture and container. Insulin quantification during storage in a TPN EVA bag has been studied but only in water [9] and aimed at illustrating adsorption on the EVA material of the bag, but not the influence of the TPN admixture. 
Various commercial kits based on immunometric methods are available on the market. The two methods compared in this study have been widely used over the last decade for insulin quantification in human serum. Interferences are one of the most notorious issues when using immunometric methods $[13,26]$. Insulin quantification is complicated in such a complex medium as TPN because of elements that can provoke disturbance between antibodies and their specific epitope. As previously described in the literature [27], additives contained in marketed insulin solutions and as supplements to the TPN medium can influence the stability of insulin in the same way as the very nature of the components of the TPN admixture can. It was therefore essential to perform this validation study in a precisely defined TPN medium. Immunometric assays seem to be by far the best analytical methods for quantifying insulin in marketed solutions. Recognition of the protein by anti-insulin antibodies should prevent interaction with additives such as m-cresol, because of the fundamental difference between the structures of insulin and m-cresol. Solutions of m-cresol and glycerol were tested with both methods to mimic the main excipients of commercially available insulins but interferences caused by these two molecules have been excluded since the signal values observed by both IRMA and IECMA were negligible (data not shown). The technical guidelines for the accreditation of validation methods in medical biology [22], published by Cofrac (French National Accreditation body) are clear enough as regards the use of commercially available kits. In the present study, the matrix of interest differs from human serum so it was necessary to make an extensive characterization of the chosen method to be able to quantify insulin in a TPN admixture. The main inconvenience was the existence of a matrix effect (mainly due to the presence of lipids that can be responsible for non-specific interference). Some solutions exist to limit the impact of the lipidic emulsion: use of Lipoclear $^{\mathrm{TM}}$ (a non ionic polymer intended for sample clarification [28]), solid phase extraction (SPE) or sample dilution. Lipoclear ${ }^{\mathrm{TM}}$ and SPE were eliminated because of 
insufficient knowledge of their impact on the structure of insulin. The diluting of initial samples was therefore selected. However, the extent of insulin immunoreactivity with immunometric methods can depend on the diluent used. All calibrators sold in commercial kits (whatever the chosen method) contain a BSA matrix (with unknown concentration) in a buffered solution: TRIS buffer for IRMA and 2-( $N$-morpholino)ethanesulfonic acid buffer for IECMA. After testing various diluents for both methods, all the measurements were finally completed after sample dilutions in 4\% BSA in PBS (pH 7.5) which yielded negligible results for both the IRMA and IECMA methods. Despite these non-zero values and considering that LOQ corresponds to about 3.3xLOD, none of the values obtained in the LOD to LOQ range could be clearly distinguished. Both the IRMA and the IECMA kits are commercialized to quantify insulin in human serum, so it seemed logical to select the diluent with the composition closest to that of human serum which contains nearly $40 \mathrm{~g} / \mathrm{L}$ of serum albumin. Sorption of insulin on the inner side of the container is a well-known phenomenon, widely described with several materials like polyvinyl chloride, polyethylene, polyurethane, or glass for example. Works carried out by Livesey et al. [29] have already underlined the importance of albumin in reducing this interaction.

The advantage of the dilution step was to provide the final sample concentration within the calibration range of the quantification kits. After this step, it was necessary to validate the methods. Accuracy profile is founded on the estimation of total errors including both systematic and random errors (absolute bias and intermediate precision standard derivation respectively). Its final representation corresponds to the largest measurement errors which seem as relevant as an individual evaluation of performance parameters and their comparison with acceptance criteria.

With this method, only IECMA was validated throughout the study. Indeed, the main argument is that the upper and lower tolerance limits calculated for each level of 
concentration fell within the $\pm 10 \%$ acceptance boundaries we had defined as targets. These tolerance levels were chosen with reference to the complexity of the TPN admixture, even though some information from previous publications suggests a limit of $\pm 20 \%$ [30]. Manufacturers' instructions specify as validation criterion: recovery has to be between 90 and 110\%. The IRMA and IECMA methods respect this condition. In such a complex medium, LOD and LOQ values calculated with either quantification method are slightly different from those given by manufacturers $(40.5 \mathrm{IU} / \mathrm{mL}$ instead of $0.2 \mathrm{IU} / \mathrm{mL}$ for LOD obtained by IECMA) although they are compatible with the concentration range chosen in this study. Finally, IECMA reduces the workload compared with IRMA (which requires lag times and protection against radioactive contamination). IECMA therefore seems preferable to IRMA for quantifying human insulin in a TPN admixture, although this method is only usable in the conditions presented in the Materials and Method paragraph: any change in the protocol would require a new validation step. In spite of their high specificity, immunometric assays can suffer from interferences and different cross-reactivities, depending on the assay (because of the nature of the included antibodies) [15]. For these reasons, routine tests have to be implemented, precisely following the same protocol as the validation one to ensure the maximum chance of success.

In conclusion, the Elecsys ${ }^{\mathrm{TM}}$ IECMA method has proved to be sufficiently precise for the quantification of human insulin in a TPN medium supplemented with vitamins and trace elements in the concentration range of 5 to $40 \mathrm{IU} / \mathrm{L}$.

\section{ACKNOWLEDGMENT}

The authors wish to thank Mrs Alexandra Tavernier, M.A. University of Glasgow, U.K.; Professeur Agrégée, University of Lille, for her extensive revision of the final manuscript. 


\section{CONFLICTS OF INTEREST}

The authors declare that they have no conflict of interest. 


\section{REFERENCES}

1. McMahon MM, Nystrom E, Braunschweig C, Miles J, Compher C, American Society for Parenteral and Enteral Nutrition (A.S.P.E.N.) Board of Directors, et al. A.S.P.E.N. clinical guidelines: nutrition support of adult patients with hyperglycemia. JPEN J. Parenter. Enteral Nutr. 2013;37:23-36.

2. Edakkanambeth Varayil J, Yadav S, Miles JM, Okano A, Kelly DG, Hurt RT, et al. Hyperglycemia During Home Parenteral Nutrition Administration in Patients Without Diabetes. JPEN J. Parenter. Enteral Nutr. 2015;

3. Olveira G, Tapia MJ, Ocón J, Cabrejas-Gómez C, Ballesteros-Pomar MD, Vidal-Casariego A, et al. Parenteral nutrition-associated hyperglycemia in non-critically ill inpatients increases the risk of in-hospital mortality (multicenter study). Diabetes Care. 2013;36:1061-6.

4. Mirtallo J, Canada T, Johnson D, Kumpf V, Petersen C, Sacks G, et al. Safe Practices for Parenteral Nutrition. J. Parenter. Enter. Nutr. 2004;28:S39-S70.

5. Bassons T, Maria Sanchez J, Bassas L, Cardona D, Ordonez J, Bonal J. Insulina recuperada según los componentes de la nutrición parenteral. Rev. SENPE. 1985;4:93-9.

6. Rusavý Z, Sramek V, Suchat R, Lacigova S, Topolcan O. Effects of carrier solution on insulin bioavailability. JPEN J. Parenter. Enteral Nutr. 2004;28:439-41.

7. Yilmaz B, Kadioglu Y, Capoglu I. Determination of insulin in humans with insulindependent diabetes mellitus patients by HPLC with diode array detection. J. Chromatogr. Sci. 2012;50:586-90.

8. Hoyer GL, Nolan PE, LeDoux JH, Moore LA. Selective stability-indicating highperformance liquid chromatographic assay for recombinant human regular insulin. J. Chromatogr. A. 1995;699:383-8.

9. Yu K-H, Tsao H-L, Lin S-J, Chen C-Y. Quantitative analysis of insulin in total parenteral nutrition bag in Taiwan. J. Food Drug Anal. 2016;24:214-9.

10. Oliva A, Fariña J, Llabrés M. Development of two high-performance liquid chromatographic methods for the analysis and characterization of insulin and its degradation products in pharmaceutical preparations. J. Chromatogr. B. Biomed. Sci. App. 2000;749:2534.

11. Hess C, Thomas A, Thevis M, Stratmann B, Quester W, Tschoepe D, et al. Simultaneous determination and validated quantification of human insulin and its synthetic analogues in human blood serum by immunoaffinity purification and liquid chromatography-mass spectrometry. Anal. Bioanal. Chem. 2012;404:1813-22.

12. Marcuard SP, Dunham B, Hobbs A, Caro JF. Availability of insulin from total parenteral nutrition solutions. JPEN J. Parenter. Enteral Nutr. 1990;14:262-4.

13. Martínez-Subiela S, Cerón JJ. Effects of hemolysis, lipemia, hyperbilirrubinemia, and anticoagulants in canine C-reactive protein, serum amyloid A, and ceruloplasmin assays. Can. Vet. J. 2005;46:625-9.

14. Owen WE, Roberts WL. Cross-reactivity of three recombinant insulin analogs with five commercial insulin immunoassays. Clin. Chem. 2004;50:257-9.

15. Heurtault B, Reix N, Meyer N, Gasser F, Wendling M-J, Ratomponirina C, et al. Extensive study of human insulin immunoassays: promises and pitfalls for insulin analogue detection and quantification. Clin. Chem. Lab. Med. 2014;52:355-62.

16. Manley SE, Stratton IM, Clark PM, Luzio SD. Comparison of 11 Human Insulin Assays: Implications for Clinical Investigation and Research. Clin. Chem. 2007;53:922-32.

17. Agin A, Jeandidier N, Gasser F, Grucker D, Sapin R. Use of insulin immunoassays in clinical studies involving rapid-acting insulin analogues: Bi-insulin IRMA preliminary assessment. Clin. Chem. Lab. Med. 2006;44:1379-82.

18. Sapin R, Le Galudec V, Gasser F, Pinget M, Grucker D. Elecsys insulin assay: free insulin 
determination and the absence of cross-reactivity with insulin lispro. Clin. Chem. 2001;47:602-5.

19. Cassidy JP, Luzio SD, Marino MT, Baughman RA. Quantification of human serum insulin concentrations in clinical pharmacokinetic or bioequivalence studies: what defines the "best method"? Clin. Chem. Lab. Med. 2012;50:663-6.

20. Levy-Marchal C, Bridel MP, Sodoyez-Goffaux F, Koch M, Tichet J, Czernichow P, et al. Superiority of radiobinding assay over ELISA for detection of IAAs in newly diagnosed type I diabetic children. Diabetes Care. 1991;14:61-3.

21. Hubert P, Nguyen-Huu J-J, Boulanger B, Chapuzet E, Chiap P, Cohen N, et al. Harmonization of strategies for the validation of quantitative analytical procedures. A SFSTP proposal--part II. J. Pharm. Biomed. Anal. 2007;45:70-81.

22. COFRAC. SH GTA 04 Guide technique d'accréditation de vérification (portée A)/validation (portée B) des méthodes en biologie médicale [Internet]. 2011 [cited 2017 Jan 10]. Available from: https://www.cofrac.fr/documentation/SH-GTA-04

23. Kelley M, DeSilva B. Key elements of bioanalytical method validation for macromolecules. AAPS J. 2007;9:E156-163.

24. Christianson MA, Schwartz MW, Suzuki N. Determinants of insulin availability in parenteral nutrition solutions. JPEN J. Parenter. Enteral Nutr. 2006;30:6-9.

25. Ciszewska M, Knyt A, Kopec B, Pertkiewicz M. Insulin availability from all in one and fat-free nutrients admixtures. Clin. Nutr. 1994;13, Supplement 1:56.

26. Sapin R. [Interferences in immunoassays: Mechanisms and outcomes in endocrinology]. Ann. Endocrinol. 2008;69:415-25.

27. Ichikawa E, Kimura M, Mori H, Yamazaki F, Hirano K. Apparent increase of insulin peak area in HPLC analysis of a preparation consisting of a mixture of insulin and total parenteral nutrition. Chem. Pharm. Bull. (Tokyo). 2006;54:1196-9.

28. Saracevic A, Nikolac N, Simundic A-M. The evaluation and comparison of consecutive high speed centrifugation and LipoClear ${ }^{\circledR}$ reagent for lipemia removal. Clin. Biochem. 2014;47:309-14.

29. Livesey JH, Donald RA. Prevention of adsorption losses during radioimmunoassay of polypeptide hormones: effectiveness of albumins, gelatin, caseins, Tween 20 and plasma. Clin. Chim. Acta Int. J. Clin. Chem. 1982;123:193-8.

30. Andersen L, Jørgensen PN, Jensen LB, Walsh D. A new insulin immunoassay specific for the rapid-acting insulin analog, insulin aspart, suitable for bioavailability, bioequivalence, and pharmacokinetic studies. Clin. Biochem. 2000;33:627-33. 
Fig. 1 Calibration standard and quality control preparation protocol. CS and QC for validation of immunometric methods were freshly prepared by spiking chosen volumes of commercial human insulin solution after dilution in $0.04 \mathrm{M}$ phosphate buffer standard (PBS) in a TPN admixture

Fig. 2 Accuracy profiles of quantification of human insulin in a TPN admixture by IRMA (2a) or IECMA (2b). The central line is the relative $\square$ bias (\%), is the relative back-calculated concentration of the validation samples, plotted with regard to their target concentration. $\diamond$ represents the $\beta$-expectation tolerance limits. External straight lines are the acceptance limits set at $\pm 10 \%$ 
Calibration standard and quality control preparation protocol. CS and QC for validation of immunometric methods were freshly prepared by spiking chosen volumes of commercial human insulin solution after dilution in $0.04 \mathrm{M}$ phosphate buffer standard (PBS) in a TPN admixture 
Accuracy profile of quantification of human insulin in a TPN admixture by IRMA (2a). The central line is the relative bias (\%), $\mathbf{-}$ is the relative back-calculated concentration of the validation samples, plotted with regard to their target concentration. Black diamond represents the $\beta$-expectation tolerance limits. External straight lines are the acceptance limits set at $\pm 10 \%$

\section{$94 \times 56 \mathrm{~mm}(600 \times 600 \mathrm{DPI})$}




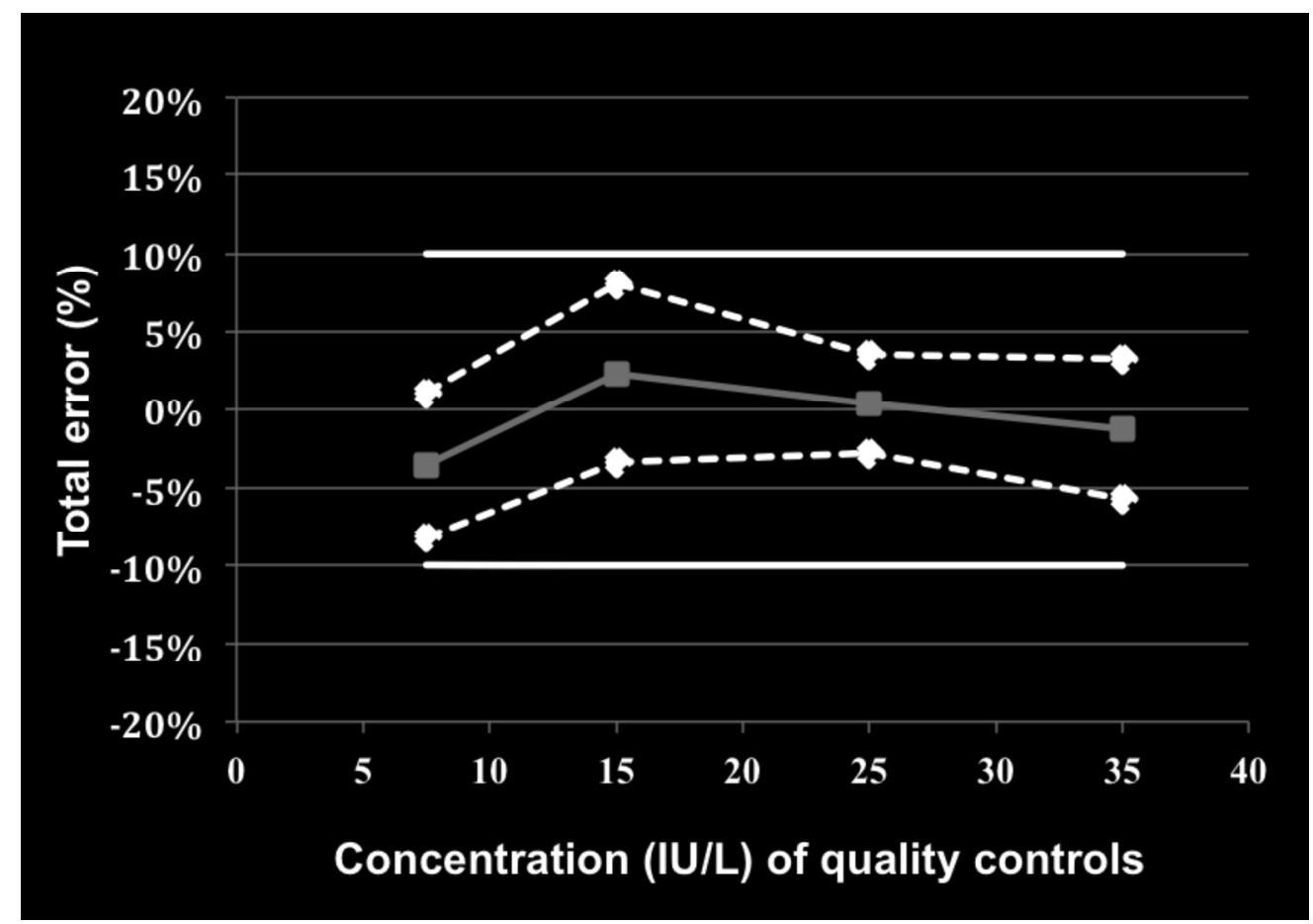

Accuracy profile of quantification of human insulin in a TPN admixture by IECMA (2b). The central line is the relative bias (\%), - is the relative back-calculated concentration of the validation samples, plotted with regard to their target concentration. Black diamond represents the $\beta$-expectation tolerance limits. External straight lines are the acceptance limits set at $\pm 10 \%$

$$
102 \times 72 \mathrm{~mm}(600 \times 600 \mathrm{DPI})
$$

